\title{
Gender, but not CYP2C19 genotypes and CYP3A phenotypes, is a major determinant of ilaprazole pharmacokinetic
}

\author{
Shan Cao ${ }^{1}$, Gan Zhou ${ }^{1}$, Yao Chen ${ }^{1}$, Dong Guo ${ }^{1}$, Zhi-rong Tan ${ }^{1}$, Lan Fan ${ }^{1}$, Hai-tang Hu ${ }^{2}$, \\ Xiang-hong Qin ${ }^{2}$, Hong-hao Zhou ${ }^{1}$, Dong-sheng Ouyang ${ }^{1}$, Wei Zhang ${ }^{1, ~}$ \\ ${ }^{1}$ Department of Clinical Pharmacology, Xingya Hospital, Central South University, Institute of Clinical Pharmacology, Hunan Key Laboratory \\ of Pharmacogenetics, Central South University, Changsha, Hunan, China \\ ${ }^{2}$ Liyzon Pharmaceutical Group Inc., Guihua Road North, Zhuhai, China
}

\section{Email address:}

Caoshan2016@163.com (Shan Cao),yjsd2003@163.com (Wei Zhang)

\section{To cite this article:}

Shan Cao, Gan Zhou, Yao Chen, Dong Guo, Zhi-rong Tan, Lan Fan, Hai-tang Hu, Xiang-hong Qin, Hong-hao Zhou, Dong-sheng Ouyang, Wei Zhang. Gender, but Not CYP2C19 Genotypes and CYP3A Phenotypes, is a Major Determinant of Ilaprazole Pharmacokinetic. American Journal of Life Sciences. Special Issue: Pharmacogenomics \& Personalized Medicine. Vol. 3, No. 1-4, 2015, pp. 14-20. doi: 10.11648/j.ajls.s.2015030104.13

\begin{abstract}
The purpose of the study was to assess the impact of CYP2C19 genotypes, CYP3A phenotypes and gender-related difference on the pharmacokinetics of new proton pump inhibitor ilaprazole. Twenty-four healthy Chinese volunteers (age $24.0 \pm$ 1.9 years) were enrolled in an open-label study stratified for gender (12 males and 12 females) and their CYP2C19 genotype (12 of $C Y P 2 C 19 * 1 / * 1$ and 12 of $C Y P 2 C 19 * 1 / * 2$ or $* 1 / * 3)$. After a single $10-\mathrm{mg}$ dose of ilaprazole was administrated, blood samples were collected at time $0,0.5,1,1.5,2,2.5,3,4,5,6,8,10,12,24,36 \mathrm{~h}$ from all subjects. Ilaprazole and its metabolite sulfone-ilaprazole plasma concentrations were measured using the well-validated HPLC/MS/MS method. CYP3A phenotype was determined by the classic CYP3A probe drug midazolam one week after the clinical trial. The kinetics characteristics of ilaprazole and sulfone-ilaprazole were significantly influenced by gender. The clearance/systemic bioavailability (CL/F) of ilaprazole was much lower in female than in male $\left(2.5 \pm 1.0\right.$ versus $\left.3.7 \pm 1.6 \mathrm{~h}^{-1}, P=0.029\right)$, difference became more significant even after corrected by body weight $(P=0.008)$. However, the differences on half-life, $\mathrm{AUC}_{0-36}$ and $\mathrm{AUC}_{0 \rightarrow \infty}$ of ilaprazole between genders were not significantly after normalized by body weight. As for sulfone ilaprazole, larger $\mathrm{AUC}_{0 \rightarrow 36}$ and $\mathrm{AUC}_{0 \rightarrow \infty}$ were detected in female when compared with male $(406.8 \pm 126.3 \mathrm{vs} .246 .7 \pm 70.0 \mathrm{ng} \cdot \mathrm{h} / \mathrm{ml}, P=0.007$, and $606.7 \pm 224.5 \mathrm{vs}$. $332.0 \pm 117.1 \mathrm{ng} \cdot \mathrm{h} / \mathrm{ml}, P=0.001)$, discrepancies were still significant after corrected by total body weight, $P$ value were 0.017 and 0.010 respectively. The pharmacokinetics parameters of ilaprazole and ilaprazole sulfone were neither different across CYP2C19 genotype groups nor related to CYP3A phenotype. CL/F of ilaprazole were much smaller in women than in men even after adjusted by body weight, indicating great effect of gender on the pharmacokinetics of ilaprazole. CYP2C19 genotypes and CYP3A phenotypes did not affect the pharmacokinetics of ilaprazole or sulfone-ilaprazole.
\end{abstract}

Keywords: CYP2C19, CYP3A Phenotype, Ilaprazole, Pharmacokinetics, Gender Difference

\section{Introduction}

Proton pump inhibitors (PPIs) are widely used in the treatment of gastric and duodenal ulcer, reflux oesophagitis and Zollinger-Ellison syndrome [1-3]. PPIs are structurally similar benzimidazole derivatives, which pharmacological effects is based on inhibition of the $\mathrm{H}^{+} / \mathrm{K}^{+}$-ATPase in the membrane of gastric parietal cells. With similar structure, newly developed drug Ilaprazole has been verified by several papers to have better therapeutic outcomes and little adverse effects [4]. However, the factor that may impact ilaprazole pharmacokinetics need to be distinguished, for so many factors, like CYP2C19 genotypes[5, 6], have been taken great clinical concern when acid-related diseases are being treated with classic PPIs.

Several studies have indicated that cytochrome P450 2C19 
(CYP2C19) is responsible for the formation of hydroxylation metabolites of omeprazole [7] and lansoprazole [8] and the demethylation metabolite of rebeprazole [9], whereas, CYP3A plays a critical role in the sulfoxidation of all three drugs using human liver microsomes in vitro [7-9]. A recent study with rats liver using on-line HPLC/ESI mass spectrometry also found two metabolites of ilaprazole, a major product, ilaprazole sulfone, and a minor product, hydroxyl-ilaprazole [10], implying that ilaprazole might be dominantly metabolized in the liver by CYP3A and partially by CYP2C19.

Recently, Cho et al [11] reported in one study only involved 4 subjects with the $C Y P 3 A 5^{*} 1 / * 1$ genotype and 3 subjects with the $C Y P 3 A 5^{*} 1 / * 3$ genotype, that $C Y P 3 A 5^{*} 3$ genetic polymorphism had no impact on the disposition of ilaprazole and sulfone metabolite, moreover, it did not correlate with intragastric $\mathrm{pH}$ elevation, which supported the finding of Li's [12], which also demonstrated no relationship between ilaprazole metabolism and CYP3A5 polymorphism in 24 subjects. However, compared with CYP3A4, CYP3A5 only plays a minor role [13], which can not substitute the total activity of CYP3A. Therefore, to obtain more accurate information, we used the classic phenotypic indicator plasma ${ }^{1} \mathrm{OH}$-hydroxymidazolam/ midazolam ratio after given 4 hours given oral midazolam $(15 \mathrm{mg})$, known as metabolic ratio (MR) to indicate the function of total "CYP3A" subfamily in both liver and intestine [14].

It has been long verified that CYP2C19 plays the most crucial role in catalyzing omeprazole and lansoprazole to form hydroxyl metabolites in the liver. In the case of ilaprazole, hydroxyl ilaprazole is minor product, so CYP2C19 is unlikely to be predominant for ilaprazole biotransformation. In addition, two previous studies have reported that CYP2C19*2 or *3 (2 common SNPs found in CYP2C19) polymorphism did not associated with ilaprazole disposition [11, 12]. However, whether ilaprazole is less influenced by CYP2C19 genotype and can be use more suitable to combination with CYP2C19 substrate like clopidogrel need to be further verified.

Clinical vital differences in therapeutic response based on gender distinction seem to be rare in pharmacokinetics, since differences of pharmacokinetics parameters will be minimized after normalizing total body weight in most cases $[15,16]$. However, significant differences in kinetics after accounting for body weight may bring on clinical divergences in the effectiveness and toxicity between genders. The US Food and Drug Administration had approved that more than 250 new drug studies for gender-related pharmacokinetics was necessary. To date, no data are available concerning the effect of gender on ilaprazole pharmacokinetics.

Present study aimed to take CYP2C19 genotypes, CYP3A phenotypes and sex factor into account to the pharmacokinetics of ilaprazole and its major metabolite sulfone ilaprazole in order to make a comprehensive understanding of ilaprazole pharmacokinetics in healthy Chinese subjects.

\section{Materials and Methods}

\subsection{Materials and Reagents}

Ilaprazole and sulfone-ilaprazole standards were obtained from the Livzon pharmaceutical Group Inc (purity: 99.1\% Zhuhai, China), ilaprazole tablets were supplied by Livzon pharmaceutical Group Inc (Zhuhai, China). Midazolam, 1OH-midazolam and internal standard propranolol and omeprazole were brought from Sigma Chemical Co. (purity: $99.0 \%$ St. Louis). Midazolam tablets were from Roche (Shanghai, China). HPLC-grade acetonitrile; methanol and methyl tert-butyl ether (MTBE) were purchased from Dikma Comp (Guangzhou, China). HPLC-grade water was from a Milli-Q system (Millipore, USA). All other reagents were of analytical grade.

\subsection{Study Subjects}

Twenty-four healthy nonsmoking Chinese subjects were enrolled in the study based on their CYP2C19 genotype that had screened previously by using the PCR-restriction fragment length polymorphism (RFLP) method, with minor modifications described by Li [12]. Due to no homozygous of CYP2C19*2 or *3 polymorphisms were found, all subjects were divided into 2 groups on the basis of CYP2C19 genotype: homozygous $(C Y P 2 C 19 * 1 / * 1)$ extensive metabolizers group (hom EMs) and $(C Y P 2 C 19 * 1 / * 3$ or $C Y P * 1 / * 2)$ heterozygous extensive metabolizers (het EMs), and each group was matched for gender, therefore, there were 6 men and 6 women in each group. This study was approved by the permission of the Xiangya institutional review boards and with the informed consent of all the subjects. Demographic characteristics of all subjects enrolled in this study are summarized in Table 1.

Subjects were healthy according to their medical history, physical examination and laboratory screening that including blood chemistries screen, liver function test, urinalysis and electrocardiogram. Exclusion criteria included any use of medication within 2 weeks prior to the commencement of the study, usage of tobacco or alcohol during the study, and clinically significant illness within 3 months before the study. A physical examination and a determination of common laboratory parameters for hematological, hepatic and renal functions were performed before, during and after the study to monitor the safety of drug treatment. Female subjects were not taking oral contraceptives and nursing or pregnant women were excluded.

\subsection{Study Protocols}

After $12 \mathrm{~h}$ fasting, all subjects received ilaprazole $10 \mathrm{mg}$ (5mg tablets, 2 pills) with $200 \mathrm{ml}$ tap water on the next morning. $8 \mathrm{ml}$ blood samples were collected before $(0 \mathrm{~min})$ and at $0.5,1,1.5,2,2.5,3,4,5,6,8,10,12,24,36 \mathrm{~h}$ after ilaprazole administration, meals is served at $4 \mathrm{~h}$ after dosing. The whole blood sample was centrifuged at $2500 \times \mathrm{g}$ for 10 minutes immediately, upper layer serums were transferred to polyethylene tubes and stored at $-80^{\circ} \mathrm{C}$ until analysis. 


\subsection{CYP3A Phenotyping}

CYP3A activity was determined for all volunteers one week after the ilaprazole was given using probe drug midazolam. The midazolam and total (unconjugated and conjugated) $1 \mathrm{OH}$-hydroxymidazolam concentrations were measured in a blood sample drawn $4 \mathrm{~h}$ after the oral administration of $15 \mathrm{mg}$ midazolam (7.5mg tablet, 2 pills), and the midazolam metabolic ratio (MR), 1OH-hydroxymidazolam/midazolam ratio, was calculated $[14,17,18]$.

\subsection{Analytic Methods}

The quantitative determination of ilaprazole and its metabolite were based on an improved LC/MS/MS method published by Zhou et al [10]. Briefly, the chromatographic condition included a Thermo HyPURITY C18 column $(150 \times 2.1 \mathrm{~mm}, 5 \mu \mathrm{m})$ with a mobile phase consisting of 10 $\mathrm{mmol} / \mathrm{L}$ ammonium formate water-acetonitrile solution (50:50, $v / v)$ at a flow rate of $0.25 \mathrm{ml} / \mathrm{min}$. The API4000 triple quadruple mass spectrometer was performed in multiple reactions monitoring mode via positive electrospray ionization interface using the transition $\mathrm{m} / \mathrm{z} 367.2 \rightarrow \mathrm{m} / \mathrm{z}$ 184.0 for ilaprazole, $\mathrm{m} / \mathrm{z} 383.3 \rightarrow \mathrm{m} / \mathrm{z} 184.1$ for ilaprazole sulfone, and $m / z \quad 346.2 \rightarrow m / z 198.0$ for omeprazole (internal standard).

The method displayed linearity over the concentration range of $0.23-2400.00 \mathrm{ng} / \mathrm{ml}$ for ilaprazole, $0.06-45.00 \mathrm{ng} / \mathrm{ml}$ for ilaprazole sulfone. The minimum limits of quantification were 0.23 and $0.05 \mathrm{ng} / \mathrm{ml}$ for ilaprazole and ilaprazole sulfone, respectively. The intra- and inter-day precisions were all less than $15 \%$ in terms of relative standard deviation (RSD), and the accuracy was within $15 \%$ in terms of relative error (RE) for ilaprazole, ilaprazole sulfone.

LC-MS equipment with ESI source and Waters Xterra $\mathrm{C}_{18}$ column $(150 \mathrm{~mm} \times 2.1 \mathrm{~mm}, 5 \mu \mathrm{m})$ were used in the experiment of midazolam concentration analysis reported previously. The column temperature was set at $40{ }^{\circ} \mathrm{C} .10 \mathrm{mmol} \cdot \mathrm{L}^{-1}$ ammonium formate-acetonitrile $(40: 60, v / v)$ was used as mobile phase and the flow rate was $0.2 \mathrm{~mL} \cdot \mathrm{min}^{-1}$. The sample room temperature was set at $5^{\circ} \mathrm{C}$ and the injection volume was $20 \mu \mathrm{L}$. Midazolam and 1-hydroxymidazolam were linear range from $0.56-287 \mathrm{ng} \cdot \mathrm{mL}^{-1}$ and $0.56-285 \mathrm{ng} \cdot \mathrm{mL}^{-1}$ respectively, the limitation of detection for midazolam and 1-hydroxymidazolam were about $0.14 \mathrm{ng} \cdot \mathrm{mL}^{-1}$ and

\section{$0.28 \mathrm{ng} \cdot \mathrm{mL}^{-1}$.}

\subsection{Pharmacokinetic Analysis}

The pharmacokinetic parameters were calculated according to a noncompartmental model using Winnonlin (Pharsight Corporation, ver.3.0, Mountain View, CA). The peak concentration $\left(C_{\max }\right)$, and the time of peak concentration $\left(T_{\max }\right)$ were directly analyzed from the visual inspection plasma concentration-time data. The elimination rate constant $(\lambda)$ were obtained by the least square fitted terminal log-linear portion of the slope of the plasma concentration-time profile, the elimination half-life $\left(t_{1 / 2}\right)$ was evaluated according to $0.693 / \lambda$. The area under the plasma concentration-time curve $\left(\mathrm{AUC}_{0-\infty}\right)$ was evaluated by the linear trapezoidal rule and further extrapolated to infinity by dividing the last measurable concentration by $\lambda$ as the equation: $\mathrm{AUC}_{0-\infty}=\mathrm{AUC}_{0-\mathrm{t}}+\mathrm{C}_{\text {last }} / \lambda$.

\subsection{Statistical Analysis}

All data are expressed as mean \pm S.D. throughout the text, tables and figures. Statistical comparisons between different groups were carried out by one-way ANOVA or non-parametric Wilcoxon signed rank sum test when there is no variance homogeneity. Simple linear correlation analysis was used to evaluate the relationship between CYP3A phenotype and pharmacokinetics parameters of ilaprazole and sulfone ilaprazole. The results were analyzed using the statistical program SPSS 13.0 for Windows (SPSS Inc, Chicago, IL). Differences were considered statistically significant at a two-tail $\mathrm{p}$ value less than 0.05 .

\section{Results}

\subsection{Effects of Gender on the Pharmacokinetics of Ilaprazole and Ilaprazole Sulfone}

Twenty-four volunteers enrolled in this study according to their CYP2C19 genotype, all of them completed the study under the well performed protocol. Mean \pm Standard deviation (SD) of height and weight were $171.6 \pm 6.4 \mathrm{~cm}$ and $63.9 \pm 7.8$ $\mathrm{kg}$ for men versus $158.1 \pm 3.9 \mathrm{~cm}$ and $51.5 \pm 4.9 \mathrm{~kg}$ for female $(P<0.0001$ for each), whereas age and BMI value did not differ between genders (shown in Table 1). No severe adverse events or clinically relevant deviations of laboratory parameters occurred among all subjects.

Table 1. Demographic characteristics of the healthy Chinese subjects enrolled in study ( $n=24)$

\begin{tabular}{lllll}
\hline Gender & CYP2C19 Genotype & Age (years) & Height (cm) & Body Weight (kg) \\
\hline Male & $1: 1(\mathrm{n}=12)$ & $21.8 \pm 2.7$ & $171.6 \pm 6.4$ & $63.9 \pm 7.8$ \\
Female & $1: 1(\mathrm{n}=12)$ & $20.5 \pm 1.7$ & $158.1 \pm 3.9^{*}$ & $51.5 \pm 4.9 *$ \\
\hline
\end{tabular}

Values are given as mean $\pm \mathrm{SD}$

$* \mathrm{P}<0.0001$

After oral administration of $10 \mathrm{mg}$ ilaprazole, ilaprazole and sulfone-ilaprazole could be detected and assessed. Plasma levels of ilaprazole and sulfone ilaprazole showed similar tendency in relation to gender difference (Fig 1). As figure showed, females had higher area under the plasma concentration-time curve than males both in ilaprazole and ilaprazole sulfone group. However, the time achieved the maximal concentration were very similar, approximately 4 hours for ilaprazole and 10 hours for ilaprazole sulfone between different genders. The pharmacokinetic parameters 
of ilaprazole and ilaprazole sulfone between male and female groups were summarized in Table 1.

For ilaprazole, the clearance/systemic bioavailability (CL/F) was much lower in female than in male $(2.5 \pm 1.0$ versus $3.7 \pm$ $\left.1.6 \mathrm{~h}^{-1}, P=0.029\right)$, the elimination half-life in female was much longer compared with the values obtained in male (5.3 \pm 0.8 versus $4.3 \pm 0.7 \mathrm{~h}, P=0.009)$. The much more amount of area under the curve is observed both in 0 to $36\left(\mathrm{AUC}_{0 \rightarrow 36}\right)$ and 0 to infinity $\left(\mathrm{AUC}_{0 \rightarrow \infty}\right)$ in female when compare with the value in the male subjects $(4569.7 \pm 1675.6$ versus $2944.5 \pm$ $895.2 \mathrm{ng} \cdot \mathrm{h} / \mathrm{ml}, P=0.016$ and $4615.4 \pm 1678.3$ versus 2994.4 $\pm 904.8 \mathrm{ng} \cdot \mathrm{h} / \mathrm{ml}, P=0.011)$. As for sulfone ilaprazole, larger $\mathrm{AUC}_{0 \rightarrow 36}$ and $\mathrm{AUC}_{0 \rightarrow \infty \mathrm{f}}$ were detected in female when compared with male $(406.8 \pm 126.3$ versus $246.7 \pm 70.0$ $\mathrm{ng} \cdot \mathrm{h} / \mathrm{ml}, P=0.007$, and $606.7 \pm 224.5$ versus $332.0 \pm 117.1$ $\mathrm{ng} \cdot \mathrm{h} / \mathrm{ml}, P=0.001)$. No other pharmacokinetics parameters were found statistically different.
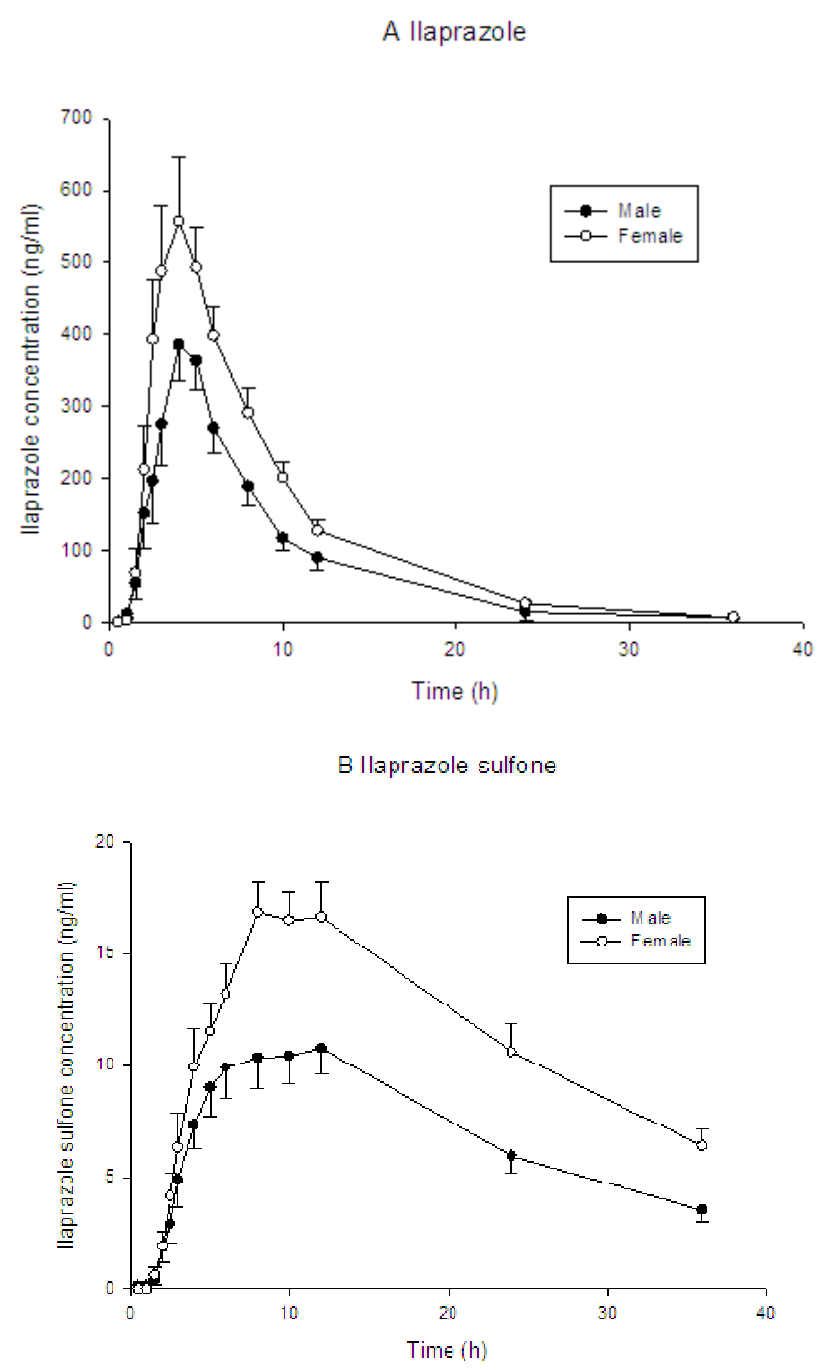

Figure 1. Plasma concentration-time profiles (Mean \pm SE) for plasma ilaprazole (A) and ilaprazole sulfone (B) after a single oral dose of $10 \mathrm{mg}$ ilaprazole in males $(\bullet)$ and females $(O)$

In attempt to exclude the weight is the factor for gender-related difference in ilaprazole pharmacokinetics, all parameters of ilaprazole and sulfone ilaprazole was adjusted by total body weight. $\mathrm{CL} / \mathrm{F}$ of ilaprazole and $\mathrm{AUC}_{0 \rightarrow 36}$ and $\mathrm{AUC}_{0 \rightarrow \infty}$ of ilaprazole sulfone still had significant difference, $P$ value were $0.008,0.017$ and 0.010 respectively.

\subsection{Effects of CYP2C19 Genotype on the Pharmacokinetics of Ilaprazole and Ilaprazole Sulfone}

No subject was found to be homozygous for the CYP2C19*2 or CYP2C19*3, therefore 24 subjects were divided into two categories, CYP2C19*1/*1 was defined as homozygous extensive metabolizers (homEMs) and CYP2C $19 * 1 / * 2$ or CYP2C19*1/*3 were classified into the heterozygous extensive metabolizers (hetEMs).

A llaprazole

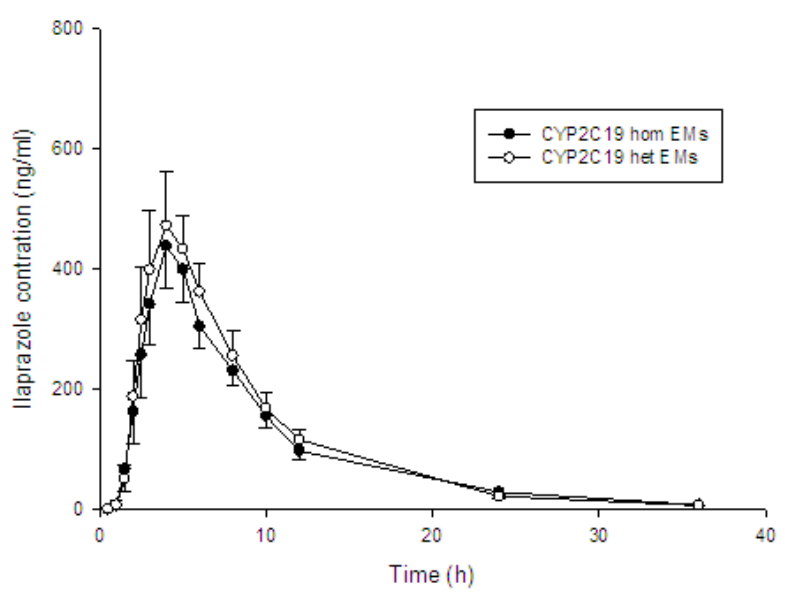

B llaprazole sulfone

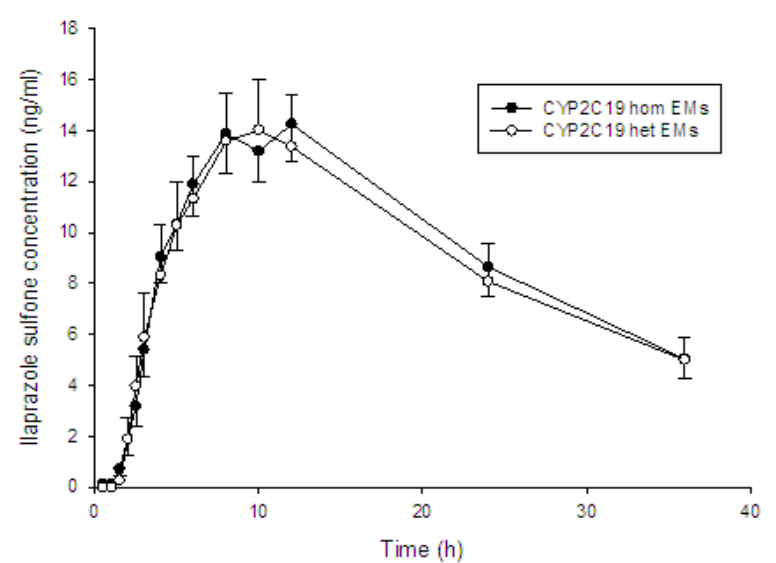

Figure 2. Plasma concentration-time curves (Mean \pm S.E.) of ilaprazole (A), ilaprazole sulfone (B) following administration of an oral 10mg ilaprazole, in 12 homo extensive metabolizer (hom EMs •) and 12 hetero extensive metabolizer (het EMs O) according to CYP2C19 genotype.

The plasma concentration-time curve of ilaprazole and ilaprazole sulfone related to CYP2C19 hom EMs and het EMs were presented in the Fig2 (A, B). The plasma concentration profile of both ilaprazole and its metabolite slightly elevated in the CYP2C19 het EMs than in hom EMs The maximal concentration of ilaprazole and ilaprazole sulfone were reached at approximately $4 \mathrm{~h}$ and $10 \mathrm{~h}$ respectively. The 
kinetics parameters of ilaprazole and ilaprazole sulfone in different CYP2C19 genotypic groups are summarized in the table 2. No parameters achieved a statistical difference.

\subsection{Effect of CYP3A Phenotype on Ilaprazole and Ilaprazole Sulfone Pharmacokinetics}

After 4 hours oral midazolam (15 mg) administration, single points of midazolam and 1-OH midazolam plasma concentration were measured. The Midazolam metabolic Ratio (MR) was calculated to evaluate collective CYP3A activity in both hepatic and intestinal. The mean $\pm \mathrm{SD}$ of the midazolam MR was $0.67 \pm 0.26$ (range 0.32-1.46) in 24 healthy subjects who were free of midazolam consumption for at least 5 days before the clinical trial. As expected, the significantly difference of midazolam MR between female and male groups have been observed $(0.82 \pm 0.28$ vs. $0.53 \pm$ $0.14, P=0.004)$, indicating that sex differences in CYP3A phenotype.

No pharmacokinetics parameters of ilaprazole and sulfone metabolite were found to be correlated with midazolam MR. To get more information about CYP3A's effect on ilaprazole pharmacokinetics, subjects were separated into 2 group, based on MRs value, which lower than 0.67 comprised the low activity group, whereas, higher than 0.67 were defined as high activity group. The subjects with lower CYP3A activity had a much higher $\mathrm{C}_{\max }$ of sulfone ilaprazole $(13.9 \pm 4.7$ vs. $18.1 \pm$ $4.9 \mathrm{ng} / \mathrm{ml}, P=0.02$ ) compared with high-activity group. No other parameters were statistically different.

\section{Discussion}

In a randomized three-way crossover study, Qiao et al [19] has successfully verified CYP2C19 genetics polymorphism is the prime factor in controlling the inter-individual variability in plasma concentration and pharmacokinetics parameters of omeprazole, lansoprazole and rabeprazole in healthy Chinese volunteers. In the three groups based on hom EMs, het EMs and PMs genotype status, the relative $\mathrm{AUC}_{0 \rightarrow \infty}$ values were 1:2.8:7.5 for omeprazole, 1:1.7:4.0 for lansoprazole and 1:1.6:3.7 for rabeprazole, respectively, indicating the lowest dependence of rabeprazole on CYP2C19 genotype during the therapeutic process. Similar findings were observed following a single oral dose of $20 \mathrm{mg}$ omeprazole, $\mathrm{AUC}_{0 \rightarrow \infty}$ values were 1:3.7:20 in 3 different CYP2C19 activity groups [20], and a single dose of $30 \mathrm{mg}$ of lansoprazole, $\mathrm{AUC}_{0 \rightarrow \infty}$ values were 1:5.7 between hom EMs and PMs [21]. However, in the present study, we found that between CYP2C19 hom EMs and het EMs groups, the relative AUC ${ }_{0 \rightarrow \infty}$ values of ilaprazole were $1: 1.2$, and CL/F were 1.2:1, which in accordance with Cho's previous study in vivo suggesting that CYP2C19 has least metabolic effect in ilaprazole [11] among PPIs. This can be possibly explained by the reason that the chemical structure of ilaprazole lacks 5-methyl group on the pyridine ring like the omeprazole, which is dependent on the metabolism by CYP2C19. Among PPIs, ilaprazole may become the drug of choice in the future due to its non-CYP2C19 elimination.

In vitro study, CYP3A was showed to play important role in ilaprazole metabolism, but until recently, no similar discovery had been made in vivo. Two studies have investigated the effect of CYP3A $5 * 3$ on kinetics profile of ilaprazole in vivo, however no relationship had been found [11, 12]. In a continuation of these earlier experiments, we use midazolam metabolic ratio as the indicator of total CYP3A activity in both hepatic and intestinal after oral administration. In our study, a relative narrow range of metabolism activity of CYP3A 4.4 fold has been found, compared with 5.9 fold interindividual variation without comedication in healthy subjects [22], moreover, the values of total MR of midazolam in our findings were (range 0.31-1.46) lower than given by Eap [22] (range 1.88-11.0), however very similar to the results represented by Link (range 0.551-1.33) after intravenous administration [23]. Expect for $C_{\max }$ of ilaprazole sulfone $(P=0.02)$, no parameters of ilaprazole or ilaprazole sulfone displayed any correlation with this CYP3A metabolic activity, even after weight adjusted (data not shown), which supposed to be non-metabolized by CYP3A in vivo and provided further evidence to previous study.

In the majority cases, when pharmacokinetics parameters were normalized by total body weight, the gender-relative differences were not evident $[24,25]$. The results of present study suggested that CL/F of ilaprazole was $33 \%$ lower in female than in male, and this difference still existed when adjusted by total body weight, which indicating body weight is not the reason for smaller $\mathrm{CL} / \mathrm{F}$ in female. Our data show that no sex-related differences in $\mathrm{V}_{\mathrm{d}} / \mathrm{F}, \mathrm{C}_{\max }$, or $\mathrm{T}_{\max }$. Due to the lower $\mathrm{CL} / \mathrm{F}$ and lack of difference in distribution, women had significantly longer elimination half-life than men. Due to the clearance differences, women had a 1.4-2 fold higher AUC than men, which strongly suggests that, at the same dosage, women are at risk for higher exposure to ilaprazole and more therapeutic efficacy than men. As illustrated in table 1, the AUC differences between female and male were not present when adjusted by total body weight, however the $\mathrm{CL} / \mathrm{F}$ was still strongly and significantly different. The reasoning for this finding is unknown.

Gender-related differences on pharmacokinetics is always a complex issue, may be involved many physiological factors and molecular mechanisms. In this study, the higher exposure of ilaprazole in female may be attributed to differences in the elimination phase, maybe related to a lower glomerular filtration rate in female than in male. The difference of intestinal protein, which may be impact transport and metabolism of ilaprazole, the hormone level and systemic availability maybe involved into the ilaprazole pharmacokinetics difference between genders as well. Clinical studies of ilaprazole in therapeutic response based on gender disparity need further investigation, and knowledge should be extended on the effects of sex on pharmacokinetics.

Although numerous studies failed to reach the agreement in gender-related effect on CYP3A activity in healthy subjects [26-29], in present study we find a statistically significant difference of midazolam MR between men and women $(0.53$ \pm 0.14 and $0.82 \pm 0.28, P=0.004)$, which has a comparably situation reported by Chen et al [30]. For midazolam is the 
substrate for both CYP3A and P-glycoprotein (P-gp), one of the most reasonable explanations of this often-observed phenomenon is that women has greater CYP3A content. Other plausible explanations involve sex steroid hormone and P-glycoprotein that lower P-gp expression in hepatic in women results in more intrahepatocelluar midazolam concentration [30]. Our study supported this observation for another occasion.

There are several limitations of this study should be mentioned. First, we did not involve the lowest CYP2C19 activity function group $(C Y P 2 C 19 * 2 / * 2$ or $C Y P 2 C 19 * 3 / * 3)$, which might result in poor persuasion. However, as the results reported by Cho, CYP2C19 het EM and PM groups had the almost overlapped plasma concentration-time curves in both ilaprazole and ilaprazole sulfone, which relatively far away from the curve of CYP2C19 hom EM group, therefore, this flaw will not likely to influence the conclusion we made. Second, the blood samples should be drawn till at least $48 \mathrm{~h}$ after dosing, for ilaprazole sulfone had a longer half-life (approximate 18 hours) than we expected. Third, ilaprazole was given orally and we had no information about its systemic bioavailability, $\mathrm{CL} / \mathrm{F}$ was expressed relative to systemic bioavailability. Further studies with intravenous ilaprazole among men and women with a wide range of body weights may determine whether our findings are results from systemic bioavailability, weight or elimination of ilaprazole.

In conclusion, gender plays an important role in ilaprazole pharmacokinetics. The pharmacokinetic characteristics of ilaprazole in healthy Chinese volunteers were not dependent on CYP2C19 genotype or CYP3A phenotype.

Table 2. Pharmacokinetics parameters of ilaprazole and metabolite sulfone-ilaprazole after a single 10 mg oral dose of ilaprazole in 12 male and 12 female subjects (mean \pm S.D.

\begin{tabular}{llll}
\hline \multirow{2}{*}{ Parameters } & Ilaprazole & & Ilaprazole sulfone \\
\cline { 2 - 4 } & Male & Female & Male \\
\hline $\mathrm{T}_{1 / 2}(\mathrm{~h})$ & $4.3 \pm 0.7$ & $5.3 \pm 0.8^{* *}$ & $15.7 \pm 4.7$ \\
$\mathrm{C}_{\max }(\mathrm{ng} / \mathrm{ml})$ & $464.5 \pm 99.6$ & $623.8 \pm 246.2$ & $12.7 \pm 4.3$ \\
$\mathrm{~T}_{\max }(\mathrm{h})$ & $3.9 \pm 1.0$ & $4.2 \pm 0.9$ & $8.8 \pm 3.3$ \\
$\mathrm{AUC}_{0 \rightarrow 36}(\mathrm{ng} \cdot \mathrm{h} / \mathrm{ml})$ & $2944.5 \pm 895.2$ & $4569.7 \pm 1675.6^{*}$ & $246.7 \pm 70.0$ \\
$\mathrm{AUC}_{0 \rightarrow \infty}(\mathrm{ng} \cdot \mathrm{h} / \mathrm{ml})$ & $2994.4 \pm 904.8$ & $4615.4 \pm 1678.3^{*}$ & $332.0 \pm 117.1$ \\
$\mathrm{~V}_{\mathrm{d}} / \mathrm{F}(\mathrm{L})$ & $22.8 \pm 8.4$ & $19.3 \pm 9.4$ & \\
$\mathrm{CL} / \mathrm{F}(1 / \mathrm{h})$ & $3.7 \pm 1.6$ & $2.5 \pm 1.0 *$ ff & \\
\hline
\end{tabular}

In note: (CYP3Aactivity-1-OH-MDZ/MDZ ratio in male group $0.53 \pm 0.14$ versus female group $0.82 \pm 0.28, P=0.004$ )

$* P<0.05, * * P<0.01$ compare with male

${ }^{£} P<0.05,{ }^{\text {£f }} P<0.01$ compare with male after adjusted by weight

Table 3. Pharmacokinetics parameters of ilaprazole and sulfone ilaprazole after a single 10mg oral dose of ilaprazole in 12 CYP2C19 hom EMs and 12 CYP2C19 het EMs (mean \pm S.D. )

\begin{tabular}{|c|c|c|c|c|}
\hline \multirow{2}{*}{ Parameters } & \multicolumn{2}{|l|}{ Ilaprazole } & \multicolumn{2}{|c|}{ Ilaprazole sulfone } \\
\hline & Male & Female & Male & Female \\
\hline $\mathrm{T}_{1 / 2}(\mathrm{~h})$ & $4.7 \pm 0.8$ & $5.0 \pm 1.0$ & $16.5 \pm 5.1$ & $19.4 \pm 8.6$ \\
\hline $\mathrm{C}_{\max }(\mathrm{ng} / \mathrm{ml})$ & $520.3 \pm 162.6$ & $572.7 \pm 239.6$ & $15.9 \pm 4.2$ & $16.0 \pm 6.2$ \\
\hline $\mathrm{T}_{\max }(\mathrm{h})$ & $4.1 \pm 0.7$ & $4.0 \pm 1.2$ & $9.3 \pm 2.6$ & $9.5 \pm 2.7$ \\
\hline $\mathrm{AUC}_{0 \rightarrow 36}(\mathrm{ng} \cdot \mathrm{h} / \mathrm{ml})$ & $3595.8 \pm 1371.4$ & $3966.9 \pm 1771.1$ & $332.3 \pm 110.7$ & $335.8 \pm 153.9$ \\
\hline $\operatorname{AUC}_{0 \rightarrow \infty}(\mathrm{ng} \cdot \mathrm{h} / \mathrm{ml})$ & $3652.9 \pm 1376.0$ & $4014.7 \pm 1772.2$ & $464.0 \pm 312.0$ & $499.6 \pm 250.8$ \\
\hline $\mathrm{V}_{\mathrm{d}} / \mathrm{F}(\mathrm{L})$ & $22.8 \pm 7.6$ & $21.2 \pm 10.0$ & & \\
\hline
\end{tabular}

\section{Statement of Interests}

This work was supported by the National Scientific Foundation of China (No. 30801421, 30901834, 30873089), Huge Project to Boost Chinese Drug Development, (No.2009ZX09501-032), 863 Project (No. 2009AA022710, 2009AA022703, 2009AA022704), New Century Excellent Talents Project (NCET-10-0843).

\section{References}

[1] Miner, P., Jr., et al., Gastric acid control with esomeprazole, lansoprazole, omeprazole, pantoprazole, and rabeprazole: a five-way crossover study. Am J Gastroenterol, 2003. 98(12): p. 2616-20.

[2] Horn, J., The proton-pump inhibitors: similarities and differences. Clin Ther, 2000. 22(3): p. 266-80; discussion 265.

[3] Robinson, M. and J. Horn, Clinical pharmacology of proton pump inhibitors: what the practising physician needs to know. Drugs, 2003. 63(24): p. 2739-54.

[4] Wang, L., et al., A new PPI, ilaprazole compared with omeprazole in the treatment of duodenal ulcer: a randomized double-blind multicenter trial. J Clin Gastroenterol, 2011. 45(4): p. 322-9.

[5] Ando, T., et al., A comparative study on endoscopic ulcer healing of omeprazole versus rabeprazole with respect to CYP2C19 genotypic differences. Dig Dis Sci, 2005. 50(9): p. $1625-31$.

[6] Tanigawara, Y., et al., CYP2C19 genotype-related efficacy of omeprazole for the treatment of infection caused by Helicobacter pylori. Clin Pharmacol Ther, 1999. 66(5): p. 528-34. 
[7] Andersson, T., et al., Identification of human liver cytochrome P450 isoforms mediating secondary omeprazole metabolism. Br J Clin Pharmacol, 1994. 37(6): p. 597-604.

[8] Pichard, L., et al., Oxidative metabolism of lansoprazole by human liver cytochromes P450. Mol Pharmacol, 1995. 47(2): p. 410-8.

[9] VandenBranden, M., et al., Interaction of human liver cytochromes P450 in vitro with LY307640, a gastric proton pump inhibitor. Pharmacogenetics, 1996. 6(1): p. 81-91.

[10] Zhou, G., et al., Identification of ilaprazole metabolites in human urine by HPLC-ESI-MS/MS and HPLC-NMR experiments. Biomed Chromatogr, 2010. 24(10): p. 1130-5.

[11] Cho, H., et al., Effect of CYP2C19 Genetic Polymorphism on Pharmacokinetics and Pharmacodynamics of a New Proton Pump Inhibitor, Ilaprazole. J Clin Pharmacol, 2011.

[12] Li, Y., et al., Pharmacokinetics of the new proton pump inhibitor ilaprazole in Chinese healthy subjects in relation to CYP3A5 and CYP2C19 genotypes. Clin Chim Acta, 2008. 391(1-2): p. 60-7.

[13] Gibbs, M.A., et al., Inhibition of cytochrome P-450 3A (CYP3A) in human intestinal and liver microsomes: comparison of Ki values and impact of CYP3A5 expression. Drug Metab Dispos, 1999. 27(2): p. 180-7.

[14] Lin, Y.S., et al., In-vivo phenotyping for CYP3A by a single-point determination of midazolam plasma concentration. Pharmacogenetics, 2001. 11(9): p. 781-91.

[15] Beierle, I., B. Meibohm, and H. Derendorf, Gender differences in pharmacokinetics and pharmacodynamics. Int J Clin Pharmacol Ther, 1999. 37(11): p. 529-47.

[16] Konieczna, L., A. Chmielewska, and H. Lamparczyk, Influence of sex on the pharmacokinetics of ciprofloxacin and ofloxacin. Chemotherapy, 2006. 52(3): p. 111-21.

[17] Thummel, K.E., et al., Use of midazolam as a human cytochrome P450 3A probe: I. In vitro-in vivo correlations in liver transplant patients. J Pharmacol Exp Ther, 1994. 271(1): p. 549-56.

[18] Thummel, K.E., et al., Use of midazolam as a human cytochrome P450 3A probe: II. Characterization of inter- and intraindividual hepatic CYP3A variability after liver transplantation. J Pharmacol Exp Ther, 1994. 271(1): p. 557-66.
[19] Qiao, H.L., et al., Pharmacokinetics of three proton pump inhibitors in Chinese subjects in relation to the CYP2C19 genotype. Eur J Clin Pharmacol, 2006. 62(2): p. 107-12.

[20] Chang, M., et al., Interphenotype differences in disposition and effect on gastrin levels of omeprazole--suitability of omeprazole as a probe for CYP2C19. Br J Clin Pharmacol, 1995. 39(5): p. 511-8.

[21] Kim, K.A., et al., Enantioselective disposition of lansoprazole in extensive and poor metabolizers of CYP2C19. Clin Pharmacol Ther, 2002. 72(1): p. 90-9.

[22] Eap, C.B., et al., Oral administration of a low dose of midazolam (75 microg) as an in vivo probe for CYP3A activity. Eur J Clin Pharmacol, 2004. 60(4): p. 237-46.

[23] Link, B., et al., Pharmacokinetics of intravenous and oral midazolam in plasma and saliva in humans: usefulness of saliva as matrix for CYP3A phenotyping. Br J Clin Pharmacol, 2008. 66(4): p. 473-84.

[24] Shimada, T., et al., Interindividual variations in human liver cytochrome P-450 enzymes involved in the oxidation of drugs, carcinogens and toxic chemicals: studies with liver microsomes of 30 Japanese and 30 Caucasians. J Pharmacol Exp Ther, 1994. 270(1): p. 414-23.

[25] Tanaka, E., Gender-related differences in pharmacokinetics and their clinical significance. J Clin Pharm Ther, 1999. 24(5): p. 339-46.

[26] Schroeder, T.J., et al., Comparison of two cyclosporine formulations in healthy volunteers: bioequivalence of the new Sang-35 formulation and Neoral. J Clin Pharmacol, 1998. 38(9): p. $807-14$.

[27] Greenblatt, D.J., et al., Age and gender effects on the pharmacokinetics and pharmacodynamics of triazolam, a cytochrome P450 3A substrate. Clin Pharmacol Ther, 2004. 76(5): p. 467-79.

[28] Lew, K.H., et al., Gender-based effects on methylprednisolone pharmacokinetics and pharmacodynamics. Clin Pharmacol Ther, 1993. 54(4): p. 402-14.

[29] Yee, G.C., et al., Age-dependent cyclosporine: pharmacokinetics in marrow transplant recipients. Clin Pharmacol Ther, 1986. 40(4): p. 438-43.

[30] Chen, M., et al., Sex differences in CYP3A activity using intravenous and oral midazolam. Clin Pharmacol Ther, 2006. 80(5): p. 531-8. 